\title{
Lengua Materna y Comunicación en la Construcción del Pensamiento Matemático
}

\section{Mother Tongue and Communication in the Construction of Mathematical Thinking}

\author{
Alfonso Jiménez Espinosa* \\ ORCID iD 0000-0001-9557-0396 \\ Ingrid Elisa Riaño Jiménez ${ }^{* *}$ \\ ORCID id 0000-0002-5463-7547
}

\begin{abstract}
Resumen
Este artículo presenta resultados de una investigación cuyo objetivo fue establecer el rol de la lengua materna en la construcción del pensamiento matemático, en un grupo multigrado de área rural. Se hace una discusión teórica sobre la comunicación, el uso de la lengua materna y las representaciones en clase de matemáticas; además, se ilustra respecto a las tareas exploratorio-investigativas de matemáticas. La investigación siguió un enfoque cualitativo, desde la perspectiva de la investigación-acción. Se describe y analiza una tarea exploratorioinvestigativa realizada con los niños, de lo cual es posible intuir que la comunicación en la clase de matemáticas a través de la lengua materna es una herramienta fundamental en el manejo concreto, conceptual o simbólico de los conceptos matemáticos. Se percibe que los niños se acercan a los significados desde su lenguaje cotidiano, asignando sentido a lo que leen, escuchan o representan a través de la interacción comunicativa oral o escrita.
\end{abstract}

Palabras clave: Comunicación. Lengua materna. Tareas exploratorio-investigativas. Matemática. Enseñanza básica.

\begin{abstract}
This article presents results of a research with the objective of establishing the mother tongue's role in the construction of mathematical thought on a multi-grade group of a rural area. There is a theoretical discussion about communication, the use of the mother tongue and representations in math class; it is also illustrated with respect to the exploratory-research mathematics task. The research followed a qualitative approach, from the perspective of action research. The exploratory-investigative task carried out with the children is described and analyzed, from which it is possible to intuit that math class communication through the mother tongue is a fundamental tool in the concrete, conceptual or symbolic handling of mathematical concepts. It is perceived that children construct meaning from their everyday language, assigning meaning to what they read, hear or represent through oral or written communicative interaction.
\end{abstract}

Keywords: Communication. Mother tongue. Exploratory-research tasks. Mathematics. Initial levels.

* Doutor em Educação, Universidade Estadual de Campinas (UNICAMP). Profesor Titular Universidad Pedagógica y Tecnológica de Colombia (UPTC), Tunja, Colombia. Dirección postal: Carrera 2 \# 40-45; Apto 403, Terrazas; Tunja, Colombia. E-mail: alfonso.jimenez@uptc.edu.co.

** Magister en Educación, Universidad Pedagógica y Tecnológica de Colombia (UPTC). Profesora Politécnico Álvaro González Santana, Sogamoso, Colombia. Dirección postal: Calle 2 \# 28 - 24, Duitama, Colombia. E-mail: iriad5@yahoo.es. 


\section{Introducción}

A pesar que la investigación en la enseñanza de las matemáticas escolares ha hecho valiosos aportes, aún es posible encontrar que el trabajo en las aulas de educación básica primaria se reduzca a una mera reproducción memorística de algoritmos y notaciones matemáticas, relegando al estudiante "el papel de receptor de información, con la única función de memorizar ideas, técnicas y procedimientos, y sin posibilidad de opinar, preguntar y mucho menos reflexionar sobre lo que debe memorizar" (JIMÉNEZ; PINEDA, 2013, p. 103).

En este proceso de maestro-emisor y estudiante-receptor, la comunicación se limita al discurso del maestro; descuida aspectos tan importantes como el tipo de lenguaje que pueden utilizar los estudiantes para representar sus raciocinios matemáticos, y la propia interacción entre estudiantes en la construcción social del conocimiento matemático (FONT, 2002).

En relación con lo anterior, es pertinente aclarar que la enseñanza de las matemáticas puede verse como una práctica social, en la que estudiantes y docentes, a través de un contexto comunicativo apropiado, pueden asignar significado y construir representaciones sobre conceptos y procedimientos de la matemática.

En la clase se utilizan, de manera explícita o implícita, unas reglas de comunicación, a partir de concepciones, de expectativas y comprensiones, respecto al acto de enseñanza y aprendizaje de las matemáticas, que son propias de la naturaleza cultural en la que se está inmerso. Parece una obviedad hacer tal precisión; sin embargo, el proceso de comunicación en la clase de matemáticas no ha tenido la suficiente importancia.

De aquí que investigar la enseñanza y el aprendizaje de la matemática desde su dimensión comunicativa puede conducir a importantes reflexiones e inquietudes sobre cuestiones de la didáctica misma. Por tal razón, en esta investigación se buscó identificar el rol de la lengua materna en la construcción del pensamiento matemático y para ello fue necesario interpretar los tipos de lenguajes utilizados por los estudiantes para comunicar y expresar procedimientos y conceptos matemáticos.

La investigación aquí presentada se sustenta en un enfoque cualitativo, desde la perspectiva de la investigación-acción y el material empírico recolectado se discute desde el lenguaje (HART, 1982; ROBITAILLE, 1992; ROMBERG, 1991; DÍAZ, 2009; DÍAZ; PALOMINO; PRIMERO, 2009), las representaciones (DUVAL, 2003, 2004; D’AMORE, 2005; HUGHES, 1987) y la comunicación en clase de matemáticas (JIMÉNEZ; SUÁREZ; GALINDO, 2010; GOÑI; PLANAS, 2011; JIMÉNEZ; PINEDA, 2013).

Además, se consideran las clases exploratorio-investigativas de matemáticas 
(FIORENTINI; CRISTOVÃO, 2006), como aquellas que priorizan el desarrollo de situaciones comunicativas, que movilizan y desencadenan tareas y actividades de pensamiento abierto, exploratorio y destacan procesos de significación matemática.

En suma, el estudio muestra un ejemplo de intervención pedagógica de cómo la comunicación de los estudiantes a través de su lengua materna - en sus diferentes representaciones - puede ser una herramienta fundamental en el manejo concreto, conceptual o simbólico de los procedimientos, notaciones y algoritmos usuales de la clase de matemáticas; pues es a partir de allí que los estudiantes discuten significados, porque este tipo de lenguaje es comprendido y aceptado por sus compañeros en la construcción del conocimiento matemático, y es natural que así sea por ser el tipo de lenguaje con el que las personas se expresan con mayor frecuencia.

\section{Discusión Teórica}

\subsection{Comunicación en la Clase de Matemáticas}

Según Goñi y Planas (2011, p. 168) "la comunicación es un proceso interactivo entre sujetos que pretenden compartir significados". Sin embargo, es necesario aclarar que la comunicación no sólo tiene que ver con el intercambio de mensajes, es importante tener presente el contexto en el que se da la acción comunicativa para asignar significado a la información que se está emitiendo, como lo afirman los mismos autores "en la acción comunicativa no se puede acceder normalmente al significado por completo sin tener en cuenta el contexto social, cultural, geográfico [...] en el que se materializa" (2011, p. 169).

En esta investigación se asumió el acto de comunicar principalmente como una forma de interacción entre individuos, con la intención de compartir, no solamente de informar (MENESES, 1999 apud JIMÉNEZ; PINEDA, 2013).

La esencia de la comunicación está en la formación de significado y esto sólo es posible gracias "a las relaciones creadas a partir de una dualidad fundamental el yo y el otro - individuogrupo, subjetividad-objetividad - llamada alteridad" (VILALBA, 2006, p. 10 apud JIMÉNEZ; PINEDA, 2013). Así mismo, los Lineamientos Curriculares conciben la comunicación como actividad fundamental para el aprendizaje y constitutiva de la comprensión de las matemáticas (COLOMBIA/MEN, 2006).

A través de la comunicación "se espera que el estudiante construya significados, reflexione, analice e intercambie interpretaciones” (JIMÉNEZ; SUÁREZ; GALINDO, 2010, p. 
179); es un proceso que ha de permitir al estudiante la confrontación de conjeturas expresadas inicialmente en su lengua materna y, posteriormente, en el lenguaje propio de la matemática.

Cabe resaltar que el importante papel de la comunicación en la clase de matemáticas, puede verse reducido a la simple transcripción de un lenguaje simbólico con carencia de sentido, inhibiendo en los estudiantes el desarrollo de su pensamiento matemático, a través de los procesos de particularizar, generalizar, conjeturar y convencer (MASON; BURTON; STACEY, 1998). Según Jiménez, Suárez y Galindo (2010, p. 180):

Con la práctica de una buena comunicación se desarrollan procesos de pensamiento donde los estudiantes son estimulados a utilizar su propio lenguaje, de tal manera que el lenguaje de la matemática surge como un proceso de construcción y no como una imposición del profesor.

De otro lado, considerar la dimensión comunicativa en el conocimiento matemático no se refiere, exclusivamente, a la representación de la realidad externa, sino a una transformación o reconstrucción de la realidad por parte del estudiante sobre los objetos en su experiencia sensible, de ahí que en el aprendizaje matemático se empleen diversos registros o representaciones matemáticas (CASTRO, 2013; JANVIER, 1987; KAPUT, 1987; RUIZ, 2000).

\subsection{Lengua materna en las clases de matemáticas}

Las matemáticas escolares no solamente se refieren a unos sistemas conceptuales organizados, al establecimiento de relaciones entre los objetos y la capacidad de operar con ellos, entre otros; sino también a su poder en el pensamiento y su relación con el lenguaje, la expresión y el simbolismo.

Es posible considerar que la matemática descansa en un "lenguaje propio, generado y pulido a través de los siglos, las culturas y los progresos técnicos, el llamado: lenguaje simbólico-matemático" (FERNÁNDEZ, 2000, p. 197). Términos como tres, decena, menor que, mayor que, arriba, son propios de la jerga matemática, que de hecho, hablando de lengua natural - materna - y de jerga matemática, dice Alcalá (2002, p. 28) "tienen una relación estrecha, la segunda depende de la primera" y esto es muy importante, pues como bien se sabe, el niño aprende a comunicarse en su lengua materna y es allí donde basa sus primeros aprendizajes.

A medida que el niño avanza en su nivel de escolaridad, su lenguaje comienza a complementarse; incluye nuevos términos como decena, centena, ángulo, cuadrado, debido al mismo paso por la escuela, y son expresiones que, si bien son tomadas de la lengua materna- 
ambiental, la matemática les asigna un significado preciso, muy peculiar (ALCALÁ, 2002).

En otras palabras, es reiterar la potencia del lenguaje como generador de actividad mental, en la construcción del pensamiento matemático, aun cuando se reconoce que en la mayoría de los salones de clase, el lenguaje, la lectura y la escritura sirven como un medio para extraer o recibir información matemática (del texto o del maestro) en lugar de ser un vehículo para articular la comprensión matemática (BORASI; SIEGEL, 1990).

En el contexto educativo de las clases de matemáticas, la lengua materna se usa para introducir temas y resumir ideas principales o hacer preguntas de comprensión (FREEMAN; FREEMAN, 2000). En otras palabras, como mencionan Genesee, Nicoladis y Paradis (1995), mientras los niños están aprendiendo matemáticas, la lengua materna es la base para involucrar un propósito comunicativo, generando motivación y comprensión, a diferencia de quienes deducen que "el aprendizaje de la matemática es independiente de la lengua natural utilizada para la enseñanza de esta disciplina, pues el lenguaje matemático es un lenguaje universal" (LIZARZABURU; ZAPATA, 2001, p. 50).

En síntesis, resulta favorable que los estudiantes de educación inicial primordialmente, utilicen su lengua materna para construir las bases de los conceptos matemáticos o de otras ciencias, pues en grados superiores el lenguaje es más descontextualizado y demanda más cognitivamente (JARRETT, 1999).

Tal como afirman Lizarzaburu y Zapata (2001, p. 189) "la lengua materna es el principal instrumento de comunicación y de desarrollo del pensamiento de una persona [...] resulta considerable el uso de la lengua materna como lengua instrumental en la enseñanza de las matemáticas".

\subsection{Las Representaciones en la Clase de Matemáticas}

Duval (2003, p. 31) sostiene que "las representaciones mentales en matemática son siempre representaciones semióticas interiorizadas en la interacción con el tratamiento de producción externa de representaciones semióticas”. Según este autor, el estudiante debe recurrir a varios registros de representación semiótica, sean gráficos, símbolos, íconos, tablas o expresiones en lenguaje natural, para lograr el aprendizaje matemático.

Duval (2004, p. 14) distingue dos conceptos fundamentales: "semiosis, la aprehensión o la producción de una representación semiótica; y noesis, los actos cognitivos como la aprehensión conceptual de un objeto". A su vez, D’Amore (2005, p. 32) establece que la necesidad de emplear diversos sistemas semióticos ligados a un mismo concepto matemático 
es absolutamente imprescindible, pues "los objetos matemáticos son abstractos y por consiguiente no manipulables como un objeto físico. Por lo tanto, no se puede acceder a ellos sin utilizar un sistema semiótico de representación".

En esencia, ningún tipo de actividad matemática puede pensarse fuera de un contexto de representación, de comunicación; de ahí la importancia de considerar las relaciones entre los diversos sistemas de representación para un mismo concepto que va más allá de la designación de un objeto.

\subsection{Clases exploratorio-investigativas}

Si se piensa en mejorar la comunicación en la clase de matemáticas, es posible considerar las clases exploratorio-investigativas (FIORENTINI; CRISTÓVÃO, 2006), como una posibilidad real de dinamizar su aprendizaje; las cuales se entienden como aquellas que movilizan y desencadenan en la clase tareas y actividades de pensamiento abierto, exploratorio y que tiene múltiples posibilidades de tratamiento alternativo y de significación.

Las clases exploratorio-investigativas designan un tipo de actividad que hace énfasis en los procesos matemáticos tales como la búsqueda de regularidades, formular, justificar y probar conjeturas, reflexionar y generalizar (PONTE et al., 1998). Estas actividades son, por lo general, para introducir un nuevo tema de estudio o para problematizar y producir significados de un concepto matemático, tal como lo expresan Fiorentini y Cristóvão (2006, p. 29):

Dependiendo de cómo se desarrollan estas clases, la actividad puede limitarse a la fase de exploraciones y problematizaciones. Sin embargo, si durante la actividad se permite la formulación de preguntas o conjeturas que desencadenen un proceso de ensayo, prueba y demostración, entonces sí se estaría hablando de la investigación de una situación matemática. Debido a esta naturaleza flexible de la tarea, clase o actividad, pueden convertirse en exploraciones o en actividades de investigación.

Desde otra perspectiva, se sabe que las clases de matemáticas pueden desarrollarse mediante la resolución de ejercicios y problemas, que tienen características que las diferencian. Un problema es una cuestión para el cual el estudiante no tiene un método de resolución inmediata (PUIG; CERDÁN 1988). Por el contrario, un ejercicio se puede resolver utilizando un método o algoritmo conocido. Sin embargo hay una característica común entre ejercicios y problemas, en ambos casos, el enunciado indica claramente lo que se da y lo que se pide. El maestro sabe de antemano que la posible resolución o respuesta del estudiante puede ser correcta o no.

Una investigación es diferente, el punto de partida es una posición abierta, o la cuestión no está completamente definida, y es allí donde el investigador (estudiante) tiene un papel clave 
en su realización (BERTINI; PASSOS, 2008). Aunado a esto último, al presentar la tarea exploratorio-investigativa es necesario que esta motive y sea desafiante, no siendo fácilmente accesible para el estudiante ni en el proceso de resolución ni en la respuesta de la actividad.

La dinámica de clase exploratorio-investigativa está influenciada por varios factores, que van desde la planificación de la tarea hasta la participación del profesor y los estudiantes en la misma. Según Ponte, Brocardo y Oliveira (2005), una clase que se desarrolle bajo el enfoque de investigación ha de ser en torno a cuatro momentos principales: introducción de la tarea (oral o escrita) y reconocimiento de la situación; formulación de conjeturas; realización de la investigación - prueba, ensayo, argumentación, demostración, finalmente, discusión de los resultados - y generalización o conclusión del trabajo realizado, donde los estudiantes exponen e informan sobre el trabajo realizado para el cierre y sistematización del conocimiento.

De acuerdo con Ponte, Brocardo y Oliveira (2005), el profesor juega un papel clave en las clases exploratorio-investigativas, debe mantener el equilibrio entre la autonomía de cada estudiante - para no poner en peligro su propia investigación - y asegurar que el trabajo del estudiante fluya naturalmente y de manera significativa.

El maestro debe interactuar con los estudiantes, teniendo en cuenta su individualidad sin descuidar la interacción entre ellos mismos. Su función es la de desafiar a los estudiantes para evaluar su progreso, su raciocinio matemático y apoyar el trabajo de los mismos, de modo que también debe tener especial cuidado en la elección de las actividades.

Nótese que este tipo de actividades en la clase de matemáticas hace hincapié en el proceso de comunicación en cada uno de sus momentos, para lo cual el contenido matemático y la lengua materna representan elementos fundamentales y complementarios que constituyen la posibilidad de conocimiento, de manera que no es conveniente considerarlos aisladamente (MACHADO, 1993).

Al respecto, Smole y Diniz (2006, p. 16) indican que en clase de matemáticas "actividades que requieren la comunicación del alumno, ayudan a esclarecer, definir y organizar sus pensamientos, haciendo que se apropien tanto de conocimientos específicos como de habilidades esenciales para aprender cualquier contenido en cualquier momento".

En las clases exploratorio-investigativas de matemáticas la lengua materna tiene dos papeles fundamentales; por un lado, los enunciados son presentados en la lengua materna usual, lo cual permite interpretar de modo preciso o aproximado la situación; de otro lado, el raciocinio matemático se apoya en el lenguaje, específicamente en la lengua materna, en su organización sintáctica y en su poder deductivo (SMOLE; DINIZ, 2006).

Esta investigación no pretendió ser exhaustiva en cuanto a la construcción de un 
concepto matemático como tal, pero sí se interesó por determinar el rol de la lengua materna en la construcción del pensamiento matemático, por tal razón se determinó que las clases exploratorio-investigativas llevarían procesos implícitos de transversalidad e interdisciplinariedad, haciendo énfasis en las tareas de identificación de patrones, regularidades o propiedades, introducción a repartos, nociones topológicas y ordinalidad.

\section{Metodología}

La investigación siguió un enfoque cualitativo, que se interesa por interpretar realidades sociales, como la del salón de clase, con la mirada y explicación de los propios sujetos involucrados en la investigación (CORBETTA, 2007).

La investigación cualitativa se interesa por el estudio de los significados e intenciones de las acciones humanas desde la perspectiva de los propios agentes sociales; es decir, se sirve de palabras, de acciones y de documentos orales y escritos para estudiar las situaciones sociales tal como son construidas por los participantes (LATORRE; RINCÓN; ARNAL, 2003).

Siguió un patrón de investigación-acción (I-A), la cual toma en cuenta al ser humano y al entorno donde se desenvuelve, vinculado con la práctica profesional, orientada a la transformación y al cambio (KEMMIS; MCTARGGART, 1992; ELLIOTT, 2005).

En al ámbito escolar la I-A se entiende como el estudio de una situación social en la que participan maestros y estudiantes con el objeto de reflexionar sobre la acción para mejorarla, para transformarla, para (re)significarla. El proceso de (re)significar se da a partir de la reflexión crítica sobre la experiencia, la construcción y reconstrucción de conocimientos en la acción, constituida por los sujetos que comparten sus conocimientos (JIMÉNEZ, 2002).

La investigación usó la observación participante (CORBETTA, 2007) junto con las notas de campo registradas durante y después de la observación; notas que atendieron a la dinámica de la clase y a los objetivos específicos de la investigación. Las notas de campo, en la concepción de Fiorentini y Lorenzato (2010, p. 119) son “uno de los instrumentos más ricos de la recolección de información durante el trabajo de campo. En estas el investigador registra observaciones de los fenómenos, hace descripciones de las personas y escenarios, describe episodios o retrata diálogos”.

Además, se emplearon registros como grabaciones de audio y vídeo (CARRILLO; MUÑOZ-CATALÁN, 2011). También fueron tenidos en cuenta las producciones escritas de los estudiantes, obtenidas en tareas de clases investigativas (FIORENTINI; CRISTOVÃO, 2006).

La investigación se adelantó en una Institución de Educación Básica del área rural de la 
región del Casanare (Colombia), con un total de dieciocho niños inscritos en los grados preescolar, primero y segundo, con edades entre los cinco y ocho años. La población escolar presenta una notoria inasistencia a la escuela, debida a que los padres de familia tienen a sus hijos como ayudantes en sus largas jornadas de trabajo del campo. El nivel educativo de los papás de estos niños apenas llega al segundo grado de básica primaria.

La investigación sigue el proceso cíclico en espiral del método de investigación-acción (ELLIOTT, 2005). El trabajo de campo se diseñó a partir de tareas exploratorio-investigativas; las clases fueron grabadas en vídeo $\mathrm{y}$, posteriormente, se transcribieron las acciones, discusiones, explicaciones y tareas realizadas por los niños y la profesora.

Además en el trabajo de campo, la observación estuvo presente en todo momento; centrándose en aspectos como la distribución de los niños en el aula, la manera en que solicitaban el turno para expresarse, las intervenciones de la docente y de cada uno de los niños, y los trabajos escritos por ellos.

Especial atención y análisis se tuvo en los momentos de discusión, conclusiones y generalizaciones de cada tarea, así como las intervenciones de cada estudiante. Es de destacar que la investigación siguió los protocolos éticos de manejo de información y conocimiento informado con los sujetos de la investigación y con los papás de los niños.

Para el análisis del material empírico se establecieron categorías, las cuales surgieron del análisis de datos cualitativos, hasta "generar afirmaciones empíricas, en gran medida a través de la inducción”; y "para establecer una evidencia probatoria”, siguiendo a Erickson (1986, p. 146).

También se hace a través de la búsqueda sistemática de la confirmación o negación de los datos. En este proceso dialéctico, se requiere "fracturar sistemáticamente los datos", para generar categorías, y “regularidades en los datos” (AGAR, 1980, p. 9). Así, en la búsqueda de los aspectos centrales del material empírico, la totalidad de los datos se examinó en profundidad y en varias ocasiones.

Las categorías establecidas son mixtas, porque se obtuvieron mediante un proceso interpretativo, directamente del material de campo al ser confrontado con la literatura (FIORENTINI; LORENZATO, 2010). Las categorías de análisis establecidas, de acuerdo al lenguaje empleado por los niños en la representación de conceptos y procedimientos matemáticos, fueron: a) lenguaje verbal oral: el niño expresa su raciocinio matemático oralmente utilizando términos de su lengua materna y lenguaje matemático; b) lenguaje verbal escrito: apoya su raciocinio matemático utilizando términos de su lengua materna y lenguaje matemático de forma escrita; c) lenguaje pictórico: emplea dibujos y grafías para representar y 
apoyar su raciocinio matemático; y d) lenguaje simbólico: emplea símbolos convencionales del lenguaje matemático para representar y apoyar su raciocinio matemático.

En cuanto al proceso de análisis, se hizo de forma transversal, pues todas las categorías se consideran simultáneamente cuando se analiza una situación o un discurso. Este tipo de análisis resulta apropiado porque las categorías no son totalmente disjuntas (FIORENTINI; LORENZATO, 2010).

\section{Resultados y Discusión}

Una de las actividades en campo comienza con la lectura los juguetes ordenados (SACRISTÁN; MUÑOZ; SALA, 2014), la cual narra la historia de un niño que luego de utilizar sus juguetes los dejaba en desorden, hasta que un día los juguetes no quisieron seguir jugando debido a la desorganización. La actividad continúa con la introducción de la tarea exploratoria para lo cual se procedió a leer una carta de la niña que había prestado los juguetes para llevarlos al salón de clase. En la carta se solicitaban sugerencias para organizar esos juguetes en una nueva habitación con un espacio muy reducido. Luego cada niño recibió un juguete y se organizaron según su preferencia para jugar por unos minutos. Mientras jugaban libremente fue posible observar cómo algunos niños intentaban colocar en cierto orden sus juguetes.

Para realizar la tarea exploratorio-investigativa los niños de preescolar manipularon cinco juguetes mientras que los estudiantes de grados primero y segundo la realizaron con diez juguetes. En la tarea exploratorio-investigativa enfocada a inspeccionar la ordinalidad, la maestra propone responder a la carta que concluía el cuento en la cual tendrían que sugerir a una niña cómo organizar sus juguetes para evitar el desorden de su habitación. A continuación, apartes de la socialización y discusión de la actividad (Figura 1):

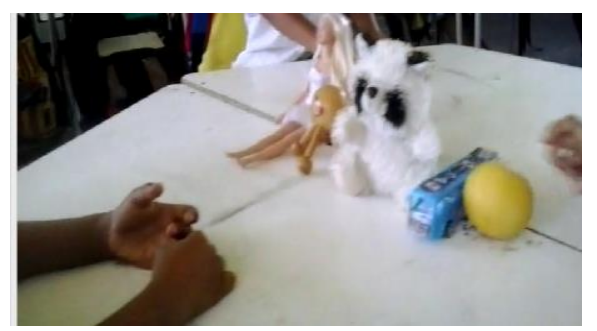

Figura 1 - Socialización de una actividad de clase Fuente: foto, material de campo

$M^{1}$ : Cuéntanos, ¿cómo lo hiciste?

E4: Los organicé aquí así (tal como se muestra en la fotografia) la muñeca va aquí adelante,

${ }^{1}$ E significa un estudiante y M, la maestra. 
aqui va esto, le sigue el oso de peluche y ahi sigue el carro y ahi sigue la pelota. (Registro de audio, 2016).

La estudiante que hace la descripción se encuentra frente a los juguetes tal como muestra la Figura 2, con una perspectiva frontal de proyección horizontal; sin embargo, ella refiere el primer objeto de izquierda a derecha y lo menciona como adelante, hasta el momento no utiliza ningún adjetivo ordinal para argumentar su representación.

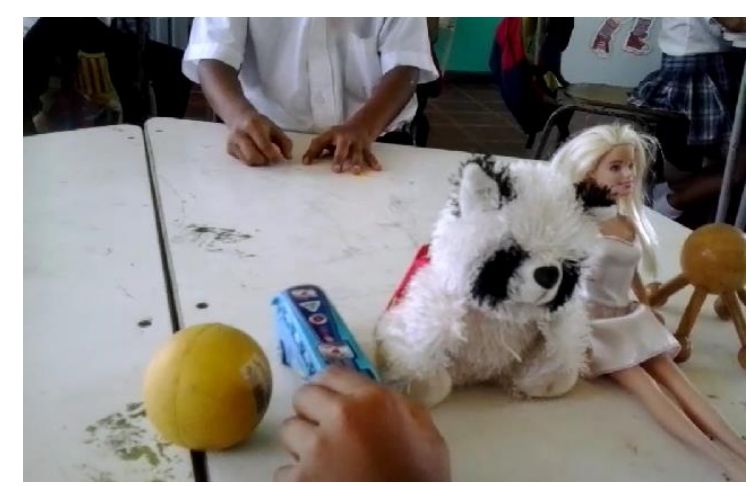

Figura 2 - Primer orden establecido a los objetos Fuente: foto, material de campo

E5: (mientras los organiza) este va aquí, este va aquí y este va aquí.

M: Podrías explicarnos ¿cómo lo hiciste?

E5: (Señalando con el dedo) la pelotaaa, el carroo...

M: ¿En qué posición está la pelota?

E5: alante [adelante] y el carro está atrás

E4: y el carro está atrás, el carro es el dos y el oso es el tres

E5: el oso alante [adelante] y la muñeca está adelante y el pulpo atrás

E4: (señalando la muñeca) eso es al pie

M: ¿la muñeca dónde está?

E4: en el cuatrorce [cuarto]

M: ¿dónde?

E4: Atrás

M: ¿Atrás de qué está la muñeca?

E6: del oso

M: ummm ¿y el pulpo en qué posición está?

E6: al pie de la muñeca.

(Registro de audio, 2016).

El propósito de la maestra era que, inicialmente, cada niño argumentara su representación individualmente; sin embargo, sus compañeros, que estaban atentos, intervinieron oralmente, generando, probablemente, la posibilidad de construcción social de conocimiento. En esta transcripción se evidencia cómo la estudiante E4 sugiere a su compañero términos de cardinalidad, pues el niño que comenzó la descripción tenía una perspectiva frontal de proyección horizontal pero no logró utilizar adjetivos ordinales para argumentarla. La niña E4 deja ver que en la numeración de posiciones tiene cierta dificultad al expresar el objeto en la cuarta posición, aunque ella sabe la secuencia verbal cardinal, hace un intento por expresar 
esa posición en ordinal, por tal razón dice cuatrorce aunque no muy segura de su intervención. Cuando la maestra pregunta de nuevo por la posición de ese objeto, ella prefiere cambiar su respuesta y dice atrás.

M: bien, organizalos tú, (dirigiéndose a otro niño), ¿cuál está de último ahi??

E6: la pelota

M: ¿y cuál está entre el carro y el osito de peluche?

Mientras E4 Y E6 señalan el carro y el osito de peluche, E5 dice: el pulpo.

(Registro de audio, 2016).

La maestra en su afán por escuchar adjetivos ordinales en las argumentaciones de sus estudiantes, comienza por hacer interrogantes con los mismos para identificar si ellos logran reconocerlos, y evidentemente, los niños reconocen adjetivos como primero, último y entre, sin embargo, hasta aquí ninguno los utilizó. Seguidamente la maestra propone que los organicen entre los tres, quedando así (Figura 3):

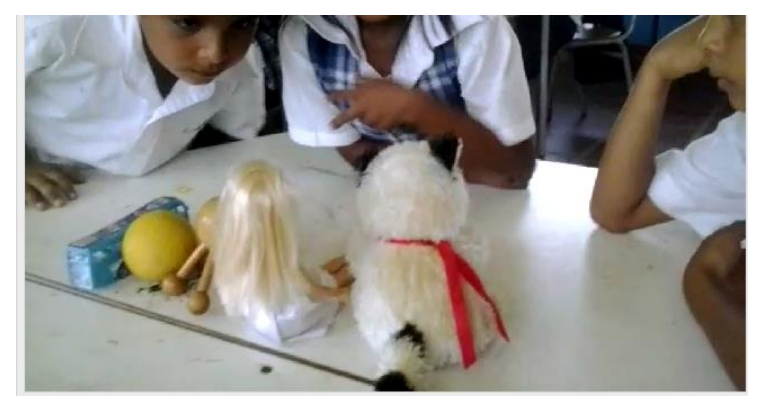

Figura 3 - Segundo ordenamiento de los objetos Fuente: foto, material de campo

M: ¿quién puede explicar cómo lo hicieron?

E4: alante [adelante] está el peluche y atrás está la muñeca y el pulpo también está atrás y este de tercero (señalando la pelota) y este de último que también está atrás.

(Registro de audio, 2016).

Al solicitar argumentación del trabajo realizado se tuvo en cuenta la posición desde la cual el sujeto hace descripción del objeto; para el caso, los niños han tenido perspectiva frontal con proyección horizontal y ellos han comenzado de izquierda a derecha según su ubicación; sin embargo, aún utilizan adjetivos como adelante y atrás, aunque ya incluyeron dos adjetivos de ordinalidad; para ellos, el primero guarda relación con adelante, y el objeto que se ubique a continuación estará atrás; desde este razonamiento lógico, el último será el de atrás teniendo en cuenta la relación entre que tiene con todos y cada uno de los demás objetos (FERNÁNDEZ, 2001). Veamos el diálogo y argumentación (Figura 4):

M: para responder la carta en una hoja, ¿cómo lo harán?

E4: (señalando cada dibujo de izquierda a derecha) acá hay un peluche y lo puse de primera y aqui una mariposa y la puse atrás, acá hay un gusano, lo puse atrás, acá hay un caballo lo puse de cuatrorce (sic) [cuarto] y una mariposa que puse atrás. 
E6: (señalando cada dibujo de izquierda a derecha) yo hice [coloqué] de primera la pelota. M: ¿de primera?

E6: de segunda

M: ¿cuál está de primera?

E4: La pelotaaa

M: ¿qué posición ocupa el gato?

E5: está en la mitaaad

E6: está en la mitad del carro (Sic)

M: ¿en la mitad del carro?

E6: está a la izquierda

M: está a la izquierda, ¿a la izquierda de qué?

E5: del carro

E6: está a la izquierda del carro

M: ¿el gato está a la izquierda del carro?

E4, E5, E6: siii (responden en coro)

E5: acá hay un gato, primero, luego un gusano, el otro primero (sic), una culebra fue el segundo

M: ¿Cuál está de primero?

E5: este, el gato

E4: ¿y cuál está de segundo?

E5: este, el gusano.

(Registro de audio, 2016).

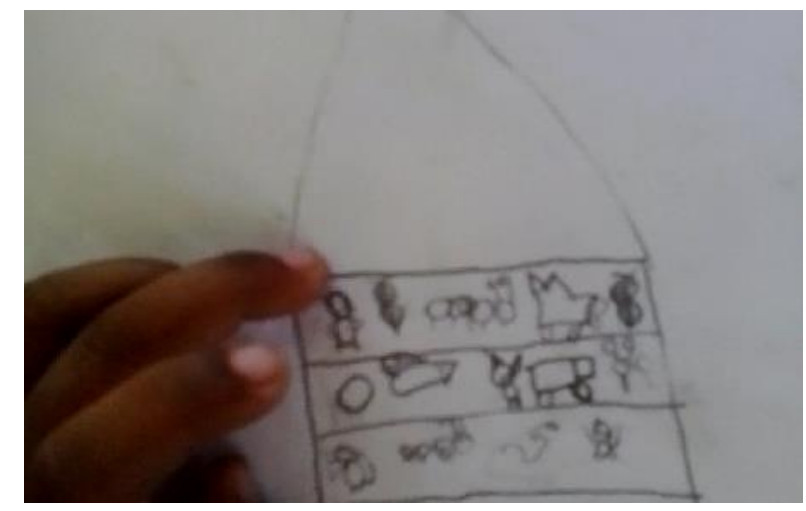

Figura 4 - Representación del orden de los juguetes Fuente: foto, material de campo

Cuando la maestra quiere constatar una respuesta para identificar que el niño tiene completamente claro qué significa su expresión, ellos prefieren cambiar su respuesta, aunque tratan de utilizar adjetivos ordinales no se sienten seguros de ello y prefieren utilizar su lenguaje informal.

Dirigiéndose a los estudiantes de grado primero, pregunta la maestra:

M: Cuéntanos, ¿cómo los organizaste?

E7: Ehhh de menor a mayor, primero el mico, primero va la licuadora, primero la princesa, primero va el carro, primero va el dinosaurio [Sic], después va la pelota, después va la computadora, después la ficha, después el pulpo y después va la ovejita.

M: listo, ¿en qué posición está la pelota?

E7: ehhh a la derecha del dinosaurio

E8: se dirige a su compañero y le dice: este es primero... segundo, tercero, cuarto, quinto, sexto, séptimo, octavo, noveno y décimo

M: Un momento, tú ¿por qué no estás de acuerdo con tu compañero?

E8: porque él dijo este primero, este primero y asi todos primero. 
M: muy bien, ahora dinos, ¿cuál juguete está entre el tercero y el quinto?

E7: ehhhh la princesa

M: ¿cuál es el tercero? Y ¿cuál es el quinto?

E7: el tercero la princesa y el quinto el dinosaurio

M: ¿Cuál está entre el tercero y el quinto?

E7: ehhh dentro del... ehh al pie... está el carro.

(Registro de audio, 2016).

Veamos la Figura 5:

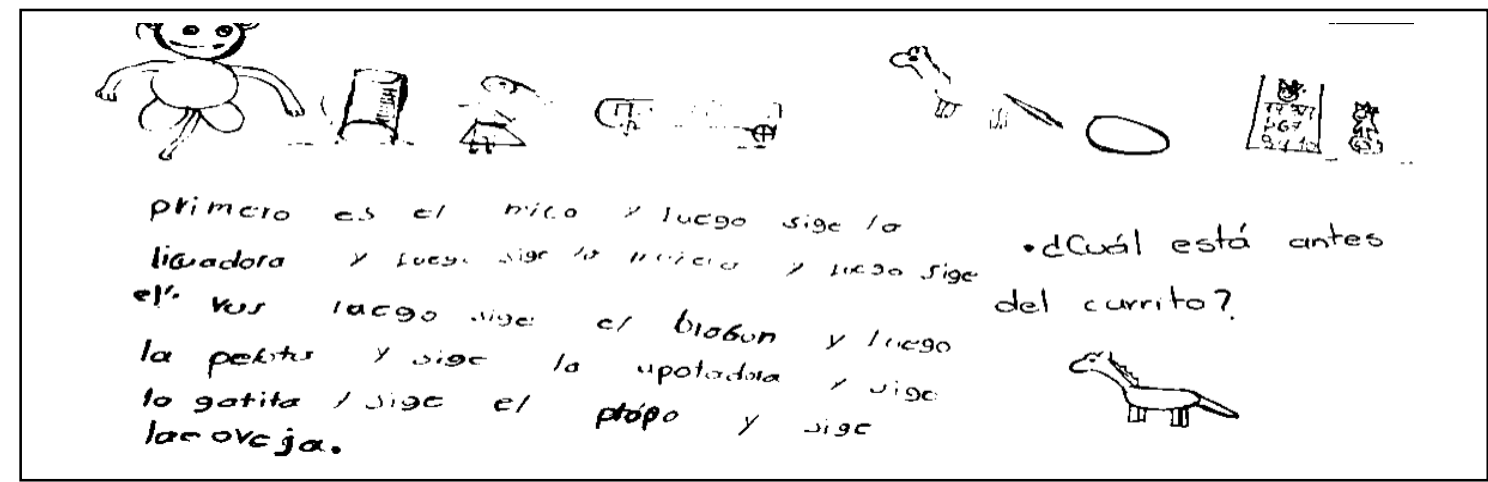

Figura 5 - Expresión escrita del orden establecido en los juguetes Fuente: foto, material de campo

El estudiante E7 comienza con los cinco primeros objetos nombrándolos como primero; a los cinco siguientes les asigna el después. Uno de sus compañeros interviene para indicarle que cada objeto se puede designar de otra forma (con un adjetivo ordinal). Nótese que cuando la maestra pregunta por la relación entre, el estudiante lo expresa y lo asimila con dentro y al pie, si bien la concepción del estudiante hace referencia a nociones topológicas, la relación entre hace referencia a una relación asimétrica y transitiva respecto a dos relaciones simultáneas, siguiente y anterior (FERNÁNDEZ, 2001).

Evidentemente, a la maestra le llama la atención cómo los niños incluyen en su descripción términos no usados por sus compañeros anteriormente; adjetivos como mayor y menor, que si bien son propios de su lengua materna, tienen su propio significado en el lenguaje matemático "tienen sentido entre números o entre medidas expresadas en las mismas unidades [...] son adjetivos que indican relaciones entre números" (D'AMORE; FANDIÑO; IORI, 2013, p. 96). Hasta aquí los estudiantes asignan los adjetivos mayor y menor respecto al tamaño del juguete (grande-pequeño).

La maestra, dirigiéndose a grado segundo: ¿cómo lo hicieron ustedes? Veamos el diálogo:

E9: lo ordenamos de menor a mayor, y le escribimos primero, segundo, tercero, cuarto, quinto... M: atentos: ¿cuál está antes del décimo?

E9: (etiquetando) primero, segundo, tercero, cuarto...

E10: ¿el noveno? Noveno, décimo. Umm ¿antes es de pa'tras? [para atrás]

E9: no hicimos más del décimo, no hay más del décimo. 
M: entonces ¿cuál está antes del décimo?

E10: el que está al revés.

M: ¿cómo es eso de que está al revés?

E9: porque no hay más

M: entonces cómo explicarían ¿cuál está antes del octavo?

E10: umm ehhh

Los niños no responden y se plantea de nuevo la pregunta

M: ¿si alguien les dice que están ubicados así: décimo, noveno, octavo, séptimo... los habrían ordenado de qué manera?

E11: al revés, de pa'tras [para atrás]

E10: de para atrás, de mayor a menor

M: bien, ¿dónde está el noveno, antes o después del décimo?

E9, E10, E11: anteees

E10: ahh ya, estaría diciendo uno antes.

(Registro de audio, 2016).

Veamos la Figura 6:

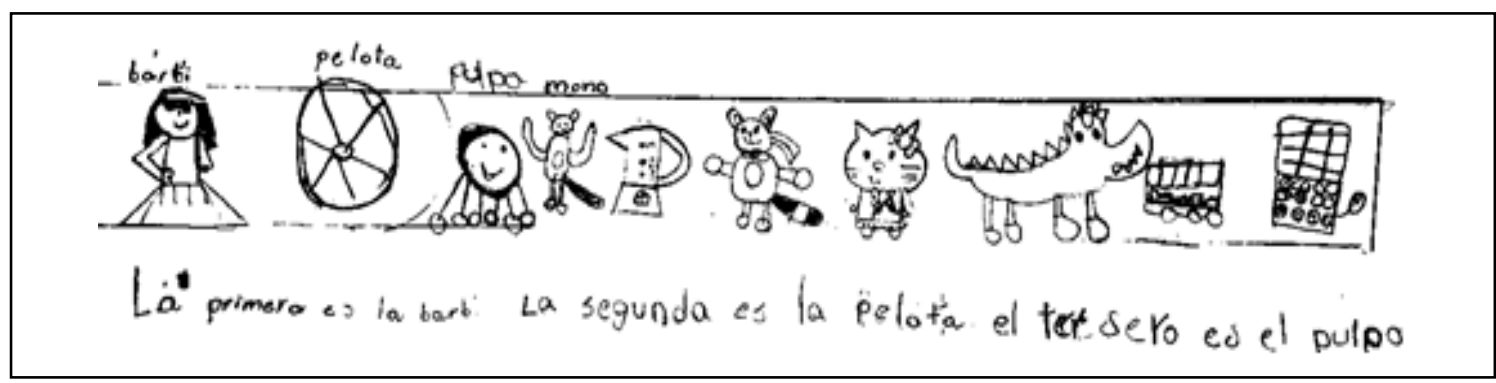

Figura 6 - Representación del orden de los juguetes y expresión escrita, hecha por un grupo Fuente: foto, material de campo

Aquí, los estudiantes no tienen muy clara la relación antes-después, lo interesante es cómo logran asimilar el antes en su lengua materna, de pa'tras [de para atrás]; sin embargo, resulta curioso cuando expresan al revés pues cuando la maestra solicita mayor argumentación, ellos reconocen que no puede ser una posición siguiente porque, para el caso, el décimo es el último objeto.

Hay que destacar, también, que los niños ante la dificultad del manejo de los ordinales (noveno y décimo), cambian el orden y toman al último (décimo) como el primero del orden inverso. La maestra replantea la pregunta solamente cambiando el ordinal y los niños no responden; no es claro aún la relación asimétrica biunívoca del anterior inmediato, por lo cual la maestra plantea una pregunta sugestiva a la relación asimétrica transitiva anterior (FERNÁNDEZ, 2001). Puede intuirse que cuando afirman al revés, los niños invierten el orden (ahora el décimo parece el primero), porque es más sencillo comenzar con los ordinales primero, segundo... que devolverse con décimo, noveno etc.

En las conclusiones y generalizaciones de la tarea se ubicó a los estudiantes de preescolar más cerca de las representaciones para que pudiesen tener mayor control del etiquetado al contar. 
M: Bien, de esta representación (mostrando una al azar), ¿cuál está entre el cuarto y el sexto?

E5, E6, E7: primero, segundo, tercero, cuarto, quinto y sexto

E10: siiii el que está en el centro es el dinosaurio.

M: muy bien, la pregunta era ¿cuál está entre ...? (sin que la maestra termine)

E10: ... el centro de todos?

Estudiantes: (en coro) el dinosaurio

M: ¿qué posición ocupa el dinosaurio?

E10: la mitad

E8: la quinta

M: bien, ¿cuál está entre el sexto y el octavo?

E9: el noveno

Estudiantes: primero, segundo, tercero.... Octavo, el séptimo

M: bien ¿cuál está antes del cuarto?

Estudiantes: primero, segundo, tercero, cuarto.... Tercero.

M: y ¿cuál está después del octavo?

Estudiantes: primero, segundo, tercero, cuarto ... octavo, noveno. El noveno.

(Registro de audio, 2016).

Si bien la intervención de todos es fundamental en la construcción del conocimiento, es de resaltar cómo entre ellos utilizan términos propios con los que se entienden mejor. Por ejemplo, la relación entre, es asociada con en la mitad y en el centro, porque el objeto que ocupa la posición de la relación en cuestión, visualmente se identifica en medio de la representación; por tal razón la maestra hace la misma pregunta, cambiando de ordinales, a lo cual los estudiantes responden haciendo uso del conteo en secuencia verbal ordinal.

Esta tarea permite identificar relaciones de ordinalidad; sin embargo, en concordancia con Fernández (2001), se evidencia que no se ha prestado la suficiente atención al respecto, pues en el currículo escolar la construcción de las relaciones de ordinalidad se da como supuesto; parece muy trivial, reduciéndolo en el aula a la memorización de algunos adjetivos ordinales y la utilización de material de copia para ejercitar aquellos adjetivos; por lo tanto, resulta interesante ver cómo los estudiantes construyen este tipo de concepto a partir de su interacción con material concreto y con la resolución de tareas investigativas que les permitan expresar su raciocinio al argumentar sus representaciones, desde su lenguaje natural.

Se puede observar la diferencia en el lenguaje de los niños, de acuerdo al grado de escolaridad. En preescolar y primero, para la ordinalidad usan más expresiones de la lengua materna, como este va aquí, este le sigue, este va al pie; mientras que ya en el grado segundo usan los ordinales y los mencionan en secuencia, pero para identificar un determinado ordinal, por ejemplo el noveno, necesitan repetir la secuencia otra vez, para concluir que es el que está antes del décimo; usando el orden inverso es de para atrás.

Para los niños de preescolar y primero, al parecer, ordenar unos elementos significa apenas que uno esté al lado de otro, bien sea atrás, adelante, en medio de, al pie o después. Lo anterior parece indicar que la sola repetición de los ordinales no significa que ya los niños 
manejan la ordinalidad, así mencionen correctamente la secuencia. Puede intuirse la importancia de explorar la lengua materna para el rastreo en esas nociones elementales de lo que Alcalá (2002) llama la jerga matemática, que se usa en lenguaje natural y lenguaje matemático, pero que a pesar de su relación estrecha, sin la primera no se llega a la segunda.

\section{Consideraciones Finales y conclusiones}

Si bien el lenguaje matemático es soporte y parte constitutiva del conocimiento matemático mismo, hay que tener en cuenta que la actividad matemática va mucho más allá de la actividad lingüística (GODINO, 2003), representacional o simbólica, como puede evidenciarse en los pasajes analizados.

La comunicación en la clase de matemáticas a través de la lengua materna puede ser, posiblemente, la principal herramienta en el manejo concreto, para pasar al conceptual y al simbólico de las notaciones y algoritmos usuales de la clase de matemáticas; pues es a partir de allí que los estudiantes tienen la posibilidad de construcción de significado.

Esto puede evidenciarse cuando la maestra pregunta: ¿cuál está antes del décimo? y un niño responde: el que está al revés. Continúan con el diálogo: - M: ¿cómo es eso de que está al revés? - E: porque no hay más. - M: ¿si alguien les dice que están ubicados así: décimo, noveno, octavo, séptimo... los habrían ordenado de qué manera? - E: al revés, de pa'tras [para atrás]. Esto se soporta en que una de las capacidades lingüísticas más importantes consiste en asignar sentido a lo que se lee, escucha o representa a través de la interacción en una situación comunicativa - oral o escrita - tal como indica el lingüista Jakobson (1978, apud PIMM, 1990, p. 81): "el lenguaje carente de significado es un sinsentido".

La comunicación verbal (oral y escrita) es una poderosa herramienta didáctica debido a que es una forma universal de interacción del ser humano, de allí que el lenguaje verbal resulta ser bastante atractivo como instrumento de comunicación de conceptos y procedimientos matemáticos; además, hace patente el pensamiento de los estudiantes, haciéndolo público, asequible a los demás, donde, bajo una discusión orientada se origina la negociación de significado, se fomenta la comunicación, prestando especial atención al argumento, procurando expresiones precisas y sucintas.

Por ejemplo, cuando la maestra dirigió unos interrogantes a una estudiante y otro de sus compañeros intervino. - M: Podrías explicarnos ¿cómo lo hiciste? - E5: (Señalando con el dedo) la pelotaaa, el carroo... - M: ¿En qué posición está la pelota? - E5: alante [adelante] y el carro está atrás. - E4: y el carro está atrás, el carro es el dos y el oso es el tres. - E5: el oso 
alante [adelante] y la muñeca está adelante y el pulpo atrás. - E4: (señalando la muñeca) eso es al pie. - M: ¿la muñeca dónde está? - E4: en el cuatrorce [cuarto] - M: ¿dónde? - E4: Atrás. Sin embargo, resultaría insuficiente reducir las clases de matemáticas a este tipo de lenguaje, pues es muy importante reconocer los diferentes tipos de representación de las ideas y conceptos matemáticos.

Para finalizar, como implicación didáctica se destaca la potencialidad de las tareas de clases exploratorio-investigativas, al ser un escenario que propicia un contexto adecuado para la comunicación y la producción de significados matemáticos a través de la argumentación y la reflexión de los estudiantes, tanto en la necesidad de expresar y explicar cómo pensaron, como para comprender a sus compañeros.

Además, estas tareas permiten valorar los diferentes tipos de lenguajes usados por los estudiantes en la representación de sus ideas, conceptos y conocimientos matemáticos. Se percibe la necesidad de seguir investigando para profundizar en la forma como los niños logran conceptualizar la ordinalidad y buscar aportes que ayuden al profesor de esos niveles a realizar su tarea.

\section{Referencias}

AGAR, M. The professional stranger: An informal introduction to ethnography. 1 edición. New York: Academic Press, 1980.

ALCALÁ, M. La construcción del lenguaje matemático. Primera edición. Barcelona. Ed Graó, 2002.

BERTINI, L. de F.; PASSOS, C. L. B. Uso da Investigação Matemática no Processo de Ensino e Aprendizagem nas Séries Iniciais do E. Fundamental. In: EBRAPEM, 12., 2008, Rio Claro. Anais... Rio Claro SP: SBEM, 2008. p. 1-17.

BORASI, R.; SIEGEL, M. Reading to learn mathematics: New connection, new questions, new challenges. For the Learning of Mathematics, v. 10, n. 3, p. 9-16, 1990.

CARRILlO, J.; MUÑOZ-CATALÁN, M. C. Análisis metodológico de las actas de la SEIEM (19972010) desde la perspectiva de los métodos cualitativos. Reflexión en torno a un caso. In: MARÍN, M. et al. (Ed.). Investigación en educación matemática XV. Ciudad Real: SEIEM, 2011. p. 77-98.

CASTRO, E. Exploración de patrones numéricos mediante configuraciones puntuales. Estudio con escolares de primer ciclo de secundaria (12-14 años). Primera edición. Granada: Comares, 2013.

COLOMBIA, MINISTERIO DE EDUCACIÓN NACIONAL (MEN). Estándares Básicos de competencias. Primera edición. Bogotá: Escribe y Edita, 2006.

CORBETTA, P. Metodología y técnicas de investigación social. Primera edición. Madrid: Mc Graw Hill, 2007.

DÍAZ, D.; PALOMINO, J.; PRIMERO, F. EI lenguaje matemático y su implicación en el aprendizaje 
de esta disciplina. 2009. 96f. Trabajo de grado (Educación matemática) - Universidad de Sucre, 2009.

DÍAZ, H. El lenguaje verbal como instrumento matemático. Educación y Educadores. Bogotá, v. 12, n. 3, p. 13-31, 2009.

DUVAL, R. Los problemas fundamentales en el aprendizaje de las matemáticas y las formas superiores del desarrollo cognitivo. Traducción de M. VEGA. Primera edición. Cali: Universidad del Valle, 2003.

DUVAL, R. Semiosis y Pensamiento humano. Registros semióticos y Aprendizajes Intelectuales. Traducción de M. VEGA. Primera edición. Cali: Universidad del Valle, 2004.

D'AMORE, B. Bases filosóficas, pedagógicas, epistemológicas y conceptuales de la Didáctica de la Matemática. Primera edición. México: Reverté, 2005.

D’AMORE, B; FANDIÑO, M; IORI, M. La semiótica en la didáctica de la matemática. Primera edición. Bogotá: Magisterio, 2013.

ELLIOTT, J. El cambio educativo desde la Investigación-Acción. Traducción de P. MANZANO. Tercera edición. Madrid: Morata, 2005.

ERICKSON, F. Qualitative methods in research on teaching. In: WITTROCK, M. C. (Ed.). Handbook of research on teaching. New York: Macmillan, 1986. p. 119-161.

FERNÁNDEZ, C. Relaciones lógicas-ordinales entre los términos de la secuencia numérica en niños de 3 a 6 años. 433f. Tesis (Doctorado en Educación) - Departamento de Didáctica de la Matemática, de las Ciencias Sociales y de las Ciencias Experimentales, Universidad de Málaga, Málaga, 2001.

FERNÁNDEZ, J. Los procesos de simbolización en matemáticas con niños ciegos y deficientes visuales. Alas para volar. In: ACTAS DEL I CONGRESO INTERNACIONAL DE NECESIDADES EDUCATIVAS ESPECIALES. Granada: Ediciones Adhara, 2000. p. 190-217.

FIORENTINI, D.; LORENZATO, S. Investigación en Educación Matemática: Recorridos teóricos e metodológicos. Tradução de A. JIMÉNEZ. Primeira edição, Campinas: Autores Associados, 2010.

FIORENTINI, D.; CRISTOVÃO, E. Histórias e Investigações de/em Aulas de Matemática. Primeira edición edição. Campinas: Alínea, 2006.

FONT, V. Una organización de los programas de investigación en Didáctica de las Matemáticas. Revista EMA, v. 7, n. 2, p. 127-170, 2002.

FREEMAN, D.; FREEMAN, Y. Preview, view, review - A useful technique. TESOL Bilingual Basics, v. 3, n. 1, p. 10-11, 2000.

GENESEE, F.; NICOLADIS, E. ; PARADIS, J. Language differentiation in early bilingual development. Journal of Child language, Cambridge, v. 22, p. 611-631, 1995.

GODINO, J. Teoría de las Funciones Semióticas. Un enfoque ontológicosemiótico de la cognición e instrucción matemática. 2003. 318 p. Tesis (Didáctica de la matemática) - Departamento de Didáctica de la Matemática, Universidad de Granada, 2003.

GOÑI, J.; PLANAS, N. Interacción comunicativa y lenguaje en clase de matemáticas. In: GOÑI, J (Coord.). Didáctica de las matemáticas. Barcelona: Editorial GRAÓ, 2011. p. 167-197. 
HART, K. Children's Understandings of Mathematics. Educational Studies in Mathematics, London: Murray, v. 13, n. 1, p. 11-16, 1982.

HUGHES, M. Los niños y los números: las dificultades en el aprendizaje de las matemáticas. Primera edición. Barcelona: Planeta, 1987.

JANVIER, C. Problems of representations in the teaching and Learning of Mathematics. Primera edición. Hillsdale, N. J: Lawrence Erlbaum Associated, 1987.

JARRETT, D. The inclusive classroom: Teaching mathematics and science to English language learners. It is just good teaching. 1 edición. Washington: Northwest Regional Educational Lab Portland, OR. Department of Education, 1999.

JIMÉNEZ, A. Quando professores de matemática da escola e da universidade se encontram: ressignificação e reciprocidade de saberes. 2002. 249 p. Tese (Doutorado em Educação) - Faculdade de Educação, Universidade Estadual de Campinas, Campinas, 2002.

JIMÉNEZ, A. ; SUÁREZ, N.; GALINDO, S. La comunicación: eje en la clase de matemáticas. Praxis \& Saber, Tunja - Colombia, v. 1, n. 2, p. 173-202, 2010.

JIMÉNEZ, A.; PINEDA, L. Comunicación y argumentación en clase de matemáticas. Educación y Ciencia, Tunja - Colombia, n. 16, p. 101 - 116, 2013.

KAPUT, J. Representation systems and mathematics. In: JANVIER, C. (Ed.). Problems of representation in the teaching and learning of mathematics. Hillsdale, NJ: Lawrence Erlbaum Associated, 1987. p. 19-26.

KEMMIS, S.; MCTARGGART, R. Cómo Planificar la Investigación-Acción. Segunda edición. Barcelona: Laerles, 1992.

LATORRE, A.; RINCÓN, D.; ARNAL, J. Bases metodológicas de la investigación educativa. Primera edición. Barcelona: Experiencia, 2003.

LIZARZABURU, A.; ZAPATA, G. Pluriculturalidad y aprendizaje de la matemática en América Latina. Experiencias y desafíos. Primera edición. Madrid: Morata, 2001.

MACHADO, N. Matemática e língua materna: análise de uma impregnação mútua. Terceira edição. São Paulo: Cortez, 1993.

MASON, J.; BURTON, L.; STACEY, K. Pensar matemáticamente. Traducción de M. Martínez. Primera edición. Barcelona: Centro de Publicaciones del MEC y Editorial Labor, 1998.

PIMM, D. El lenguaje matemático en el aula. Primera edición. Madrid: Ed Morata, 1990.

PONTE, J. P.; BROCARDO, J.; OLIVEIRA, H. Investigações matemáticas na sala de aula. Segunda edición. Belo Horizonte: Autêntica, 2005.

PONTE, J. P. et al. Histórias de investigações matemáticas. Primeira edición. Lisboa: Instituto de Inovação Educacional, 1998.

PUIG, L.; CERDÁN, F. Problemas aritméticos escolares. Primera edición. Madrid: Síntesis, 1988.

ROBITAILLE, D. National contexts for mathematics and science education. An encyclopedia of the education systems participating in TIMSS (pp. 82-90). Vancouver: Pacific Educational Press, 1992. 
ROMBERG, T. A. Características problemáticas del currículo escolar de matemáticas. Revista de Educación, Madrid, v. 294, p. 323-406, 1991.

RUIZ, F. La tabla-100: representaciones geométricas de relaciones numéricas. Un estudio con profesores de primaria en formación. 440f. Tesis (Doctorado en Didáctica) - Universidad de Granada, Granada, 2000.

SACRISTÁN, P; MUÑOZ, J; SALA, F. Los juguetes ordenados. 1 edición Madrid: Cuentos para dormir, 2014.

SMOLE, K; DINIZ, M. Ler, escrever y resolver problemas. Habilidades básicas para aprender matemáticas. Primeira edição. São Paulo: Armted Editora S. A., 2006.

Submetido em 21 de Fevereiro de 2018. Aprovado em 24 de Julho de 2018. 